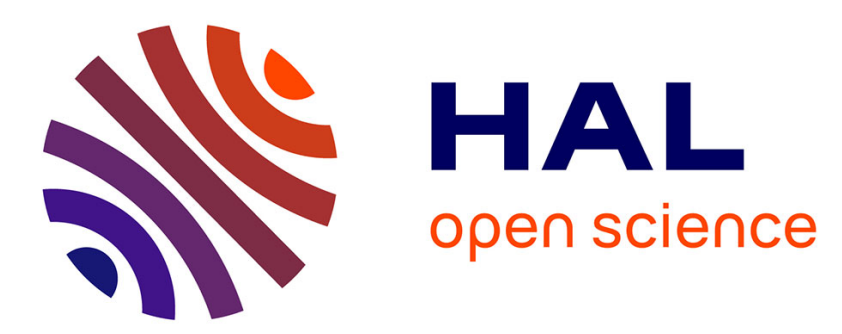

\title{
Automatic analysis and characterization of the hummingbird wings motion using dense optical flow features
}

Fabio Martínez, Antoine Manzanera, Eduardo Romero

\section{To cite this version:}

Fabio Martínez, Antoine Manzanera, Eduardo Romero. Automatic analysis and characterization of the hummingbird wings motion using dense optical flow features. Bioinspiration and Biomimetics, $2015,10.1088 / 1748-3190 / 10 / 1 / 016006$. hal-01245084

\author{
HAL Id: hal-01245084 \\ https://hal.science/hal-01245084
}

Submitted on 16 Dec 2015

HAL is a multi-disciplinary open access archive for the deposit and dissemination of scientific research documents, whether they are published or not. The documents may come from teaching and research institutions in France or abroad, or from public or private research centers.
L'archive ouverte pluridisciplinaire HAL, est destinée au dépôt et à la diffusion de documents scientifiques de niveau recherche, publiés ou non, émanant des établissements d'enseignement et de recherche français ou étrangers, des laboratoires publics ou privés. 


\title{
Automatic Analysis and Characterization of the hummingbird wings motion using dense optical flow features
}

\author{
Fabio Martínez ${ }^{1}$, Antoine Manzanera ${ }^{2}$, Eduardo Romero ${ }^{1}$ \\ ${ }^{1}$ Computer Imaging and Medical Applications Laboratory - CIM@LAB, Universidad \\ Nacional de Colombia \\ ${ }^{2}$ Unité d'Informatique et d'Ingénierie des Systèmes, ENSTA-ParisTech \\ E-mail: edromero@unal.edu.co
}

\begin{abstract}
A new method for automatic analysis and characterization of recorded hummingbird wing motion is proposed. The method starts by computing a multiscale dense optical flow field, which is used to segment the wings, i.e., pixels with larger velocities. Then, the kinematic and deformation of the wings were characterized as a temporal set of global and local measures: a global angular acceleration as a time function of each wing and a local acceleration profile that approximates the dynamics of the different wing segments. Additionally, the variance of the apparent velocity orientation estimates those wing foci with larger deformation. Finally a local measure of the orientation highlights those regions with maximal deformation. The approach was evaluated in a total of 91 flight cycles, captured using three different setups. The proposed measures follow the yaw turn hummingbird flight dynamics, with a strong correlation of all computed paths, reporting a standard deviation of $0.31 \mathrm{rad} / \mathrm{frame}^{2}$ and $1.9(\mathrm{rad} / \mathrm{frame})^{2}$ for the global angular acceleration and the global wing deformation respectively.
\end{abstract}

Keywords: wing hummingbird flight, dense optical flow, hummingbird yaw turn analysis, Kinematic hummingbird patterns

Submitted to: Phys. Med. Biol. 


\section{Introduction}

Emulation of flying animal movements may facilitate the design of smarter micro air vehicles (MAVs), allowing them to perform more complex maneuvers and expend less energy [1]. Among these MAVs, hovering machines have succeeded in executing elaborated movements within very limited spaces [1]. Designs of such new hovering MAVs may be inspired in animals such as the Hummingbird [2] [43], a bird species able to hover in mid-air and flight backwards [44]. Their maneuverability is based on their ability to easily turn in very restricted spaces, a skill developed to access flowers with sugar rich nectars textcolorMyColor and also to catch insects. A proper design of flapping-wing flight machines requires a precise comprehension of the many interactions between kinematic and morphological wing parameters. Temporal wing deformation patterns constitute the fundamental variables to estimate the nonlinear relationships between the different wing segments [18]. Moreover, rotations and velocities are related not only with kinematic information but also with forces and torques produced by muscles and tendons [3], [1]. Nevertheless, in spite of extensive studies, many of the morphological, local dynamic, kinematic and even aerodynamic details are poorly understood [1] because of the still limited tools used to capture flight patterns, like for instance flapping frequency, stroke amplitude, angular velocity and even spatio-temporal wing deformation.

Many questions about the flight mechanisms still remain open, e.g., how the hummingbird accomplishes yaw turns during the hovering flight or how the bird is able to keep turning once he starts this maneuver. Altshuler et al. [4], have recently published a study about the wingbeat kinematics and the motor control of yaw turns. This investigation correlated muscle EMG signals with kinematic trajectories and found differences among the different stroke flight phases and even asymmetry between the wings. In many cases however, the only available information about these turns comes from other species like the gliding or flight patterns observed in insects [5], [6], [7], [8], [10], [11]. However these animals are actually very different since they are entailed with simple wings that operate over a larger range of speeds [9] and are supported by an outer skeleton. In addition, while birds roll their body to orient the wing forces, insects can easily turn by asymmetric left-right wingbeats [4], [45], [34], [35] . The hummingbird flight has been broadly studied using customized analysis systems that require a set of reference points, somehow highlighted and superimposed on the animal structure. These salient anatomical points are usually drawn by an expert [12], adding new variables to the analysis since such delineation is a frame-per-frame task which inevitably leads to high inter and intraobserver variabilities. Smoothing and filtering strategies are often used to remove errors introduced during the digitazing process[46]. However, these strategies are sensible to the parameters of starting and require a fully knowledge about the signal nature, for instance, a higher sample rate allows the trade off of the signal. Any modification of the signal can lead to an alteration of the observational data. Furthermore, these tools introduce artifacts that alter the natural hummingbird motion gestures since they need markers that must be placed on the wing bird, markers that in addition can be easily occluded during a wing cycle [13]. Currently, a pyramidal Lukas-Kanade optical flow strategy 
have been proposed to estimate the deformation of birlike airfoil showing coherent results by using artificial structures. This strategy is however limited to follow large displacements since the optical flow strategy used is based on a local regularization, thereby assuming that the object has small displacement among consecutive frames [47]. .

Classical approaches may miss many important details of the real dynamic patterns since they rely completely in both an expert user and an invasive tracking strategy. Recently, some approximations have automatically estimated the dynamics of flight trajectories, most of them using machine-learning methods that require a considerable number of videos for training and finding global flight patterns [14]. Overall, these kinematic methods model the wing as a stiff airfoil [24], but in reality the wing is a very flexible structure, able to asymmetrically produce non uniform movements [24]. Some works have extracted patterns to characterize the hovering hummingbird dynamics [15], supporting the importance of performing analyses of the whole structure rather than following some isolated reference points. A very useful tool has been the Particle image velocimetry (PIV) [16],[17], a strategy that permits to estimate aerodynamic variables by indirect measurement of the resultant flow produced in the air by the wing motion. In practice the set of particles, near the wing boundary, is supposed to closely follow the animal motion. The whole procedure consists then in illuminating such particles with a pulsed light laser and capturing this motion with a camera. A flow field is obtained by cross-correlating consecutive image patterns formed by particles highlighted by the laser[16], [17]. A main advantage of this method is its relative non-invasiveness, but a limitation is that the particle illumination may produce interference phenomena near the wing boundary. Besides, the whole dynamics can not be estimated with the PIV method since the captured flow is related to the external motion and hence the analysis of forces and torques is only indirect. In other words, this method may improve understanding of the hummingbird movement from an aerodynamic standpoint, but not from a kinematic point of view, whose importance is mostly associated to the local wing deformation and temporal rotation. Lately, several researchers have introduced mechanical and mathematical wing models, aiming to exploit some basic physical relationships to simulate different types of wing motions [19], [20], [21], [22]. However, these models are very restrictive, for instance wings are represented as rigid structures and therefore only very linear approximations are possible, whereby the obtained conclusions are limited and the predictive model capabilities are largely reduced [23].

In this work, a novel description of the wing dynamics is introduced, providing previously unavailable information about rotational and deformation wing patterns. This spatiotemporal description is based on a set of velocity vectors obtained from a dense optical flow, computed from sequences captured with a 2D camera which is located perpendicular to the main axis of the bird. Four principal temporal estimations are computed: (1) the global angular acceleration of the hummingbird, (2) the angular acceleration profiles computed over different wing segments, (3) the variance of the spatial orientation of the velocity of the optical flow field for each of the two wings and (4) a distribution map of the local orientation variation within the wing. These four complementary measures allow not only to follow up the wing but also to determine the instants of maximal and minimal deformations, as well as the regions 
with larger deformation. This paper is organized as follows: Section 2 introduces the proposed method, section 2.4 describes the dataset, section 3 demonstrates the effectiveness of the method and section 4 presents a discussion of the proposed work. The last section concludes and presents some possible future works.

\section{Materials and Methods}

Overall, a dense optical flow provides a motion description by estimating the instantaneous displacement of each pixel between consecutive frames. Such vector of velocity optical flow allows to compute four complementary motion descriptors at each time step, all of them further described hereafter.

\subsection{The Dense Optical Flow Computation}

First, a dense optical flow is estimated by matching all the points from frame to frame, using a nearest neighbor search in a projected feature space [25]. The multidimensional feature space is herein obtained using the local jets [27], an approximation to the image spatial structure provided by derivatives at different orders and scales: $f_{i j}^{\sigma}=f * \frac{\partial^{i+j} G_{\sigma}}{\partial x_{1}^{i} \partial x_{2}^{j}}$, where $G_{\sigma}$ is the 2 d Gaussian function with standard deviation $\sigma$. Each pixel is associated then to a feature vector defined as the collection $\left\{f_{i j}^{\sigma} ; i+j \leq r, \sigma \in S\right\}$, where $r$ is the derivation order and $S=\left\{\sigma_{1}, \ldots \sigma_{q}\right\}$ the selected scales. The set of features, associated to a frame, is represented in a kd-tree structure which facilitates efficient distance search [26]. Finally $\mathbf{V}_{t}(\mathbf{x})$, the apparent velocity vector for pixel $\mathbf{x}$ at time $t$ is calculated by coupling the feature vector associated to $\mathbf{x}$ to its nearest neighbor in the feature space calculated at time $t+1$. Thanks to the multiscale representation, which implies the matching of that extended spatial structure, this method provides a spatially consistent dense optical flow, without using explicit spatial regularization as required in other methods [38], [37].

\subsection{Hummingbird motion patterns}

The quantified optical flow is not altered by the hummingbird spinning (at $\sim 30: H z)$, basically because this movement is parallel to the camera axis and therefore the optical flow is unable to capture, i.e., only those particles moving in the plane perpendicular to the camera can be tracked. Quantifying kinematic flight patterns and their structural relations is crucial for optimal design and development of flapping-wing flight prototypes. These complex relationships may be primarily determined from the analysis of variables like the angular acceleration and temporal wing deformation, herein estimated from the dense optical flow. Such variables are temporal functions, some of them being global scalar functions of $t$ and other local spatial maps indexed by t. Local dynamic patterns are determined by firstly segmenting the calculated apparent velocity flow, finding the two largest connected regions that correspond to the right and left wing and denoted $\mathbf{V}_{\mathbf{r} t}$ and $\mathbf{V}_{1 t}$. The isolated remaining flow areas are assigned to the nearest region, according to the spatial Euclidean distance. 


\subsection{Angular Acceleration patterns}

Rotational wing patterns are crucial to understand complex flight mechanisms, like: an optimal angle of attack, a large downstroke span ratio and a high wingbeat frequency that together maintain the achieved equilibrium and facilitate complex maneuvers [28], [29]. In this work, the angular acceleration was estimated by associating the orientation of the computed apparent velocity optical flow and the wing, under the assumption that the rotation axis is perpendicular to the optical axis of the camera [30], [31]. Two configurations were herein proposed to analyze the hummingbird wing motion from an angular acceleration standpoint: a global angular acceleration of both wings and a temporal map of regional angular accelerations, constructed by estimating the angular acceleration profiles for a set of wing segments which correspond to a wing anatomical partition.

The Global Angular acceleration for each wing: For a non-zero flow vector $\mathbf{V}$, let $\phi(\mathbf{V})$ be the apparent velocity flow orientation, the wing flows $\mathbf{V}_{\mathbf{t}}^{\mathbf{r}}$ and $\mathbf{V}_{\mathbf{t}}^{\mathbf{l}}$ were followed, corresponding to the right and left wings, which are bounded within a bounding-box at a particular time $t$ and oriented to the main direction of each wing.Then, an averaged spatial orientation for each wing $\overline{\phi\left(\mathbf{V}^{r, l}\right)_{t}}$ is calculated as the linear combination of every orientation, weighted by its norm. Under the assumption that there exists a dominant orientation of the velocity field, the rate of change of the angular velocity is then computed as $\Omega_{t}=\frac{d \overline{\phi\left(\mathbf{V}^{r, l}\right)_{t}}}{d t}$. This variable describes the turn angle during the flight, and highlights the relaxation and effort phases.

Angular acceleration spatial profiles: An angular acceleration profile was herein obtained Hence, left and right temporal series are constructed by associating a wing region to the closer region in the precedent frame, in terms of the minimal distance $d_{t+1}^{r, l}$ between the centroids of consecutive wing regions $(\bar{x}, \bar{y})_{\mathbf{s}_{\mathbf{t}}^{\mathbf{r}}, \mathbf{l}}$, as follows: $d_{t+1}^{r, l}=\left\|(\bar{x}, \bar{y})_{\mathbf{s}_{\mathbf{t}}^{\mathbf{r}, \mathbf{l}}}-(\overline{x y})_{\mathbf{s}_{\mathbf{t}+\mathbf{1}}^{\mathbf{r}, \mathbf{l}}}\right\|_{2}^{2} \cdot \quad$ Then, a local angular estimation was herein obtained from the temporal acceleration profile of $\mathbf{s}_{\mathbf{t}}{ }^{r, l}$ by dividing the whole region into a set of $N$ segments perpendicular to the main direction, whereby $\mathbf{s}_{\mathbf{t}}{ }^{r, l} \in$ $\left\{s^{\{r, l\}_{0}}, s^{\{r, l\}_{1}}, \ldots, s^{\{r, l\}_{i}}, \ldots, s^{\{r, l\}_{N-1}}\right\}_{t}$ (Figure 1 (d)). Finally, a local angular acceleration for the $i-t h$ segment $s_{t}^{i}$ was computed and its temporal map $\Omega\left(s_{i}\right)$ was obtained. Such representation is:

$$
\Omega\left(s_{i}(\mathbf{x}, t)\right)=\left\{\frac{d \overline{\left.\phi\left(\mathbf{V}_{t}(\mathbf{x})\right)\right)}}{d t} ; x: \mathbf{x} \in s_{i}\right\}
$$

2.3.1. Wing deformation patterns Although hummingbird kinematic patterns are generally similar to the insect flight, the morphological structure of the wings are very different given the mass and structural relationship, furthermore if considering that the wing muscle mass is largely determined by the need of producing efficient mechanical power. This is why the impact of the wing deformation during the flight must be quantified for understanding how the wing geometry supports forces and produces 
motion. For instance, it has been documented [15] that the hummingbird upstroke and downstroke are not symmetrical. Such observation is strongly related to the local loads supported along the wing.

Wing deformation is herein estimated by computing the variance of the local velocity along the time. This estimation is carried out by assuming that every flow vector should be equally distributed along a rigid wing, while deformable structures produce a non homogeneous distribution, whereby the local motion variance is proportional to the physical deformation.

Local map of the wing deformation: The wing flow and its averaged orientation are used to compute a per-frame deformation map, estimated as a squared difference between each non-zero flow vector and the average orientation:

$$
Q_{t}^{l, r}(\mathbf{x})=\left\|\phi\left(\mathbf{V}_{t}(\mathbf{x})\right)-O_{t}^{l, r}\right\|_{2}^{2}
$$

This analysis provides a stress map of the wing, highlighting the forces shearing the wing 1 .

Global wing deformation: For each wing, a global deformation $W_{t}^{l, r}$ is estimated $\ddagger$ by averaging the local wing deformation map $Q_{t}^{l, r}(\mathbf{x})$. This measure allows to detect the largest deformation instants during a flight stroke.

\subsection{Dataset description}

Four annas hummingbirds (Calypte Anna's) were recorded. Each hummingbird was placed inside a flight chamber that contained a wooden perch in the corner and a feeder, in which a $1 \mathrm{ml}$ syringe was mounted at the end of a metallic arm $(0.22 \mathrm{~m} \times 0.19 \mathrm{~m} \times 0.15 \mathrm{~m})$. The feeder arm was connected to a stepper motor (MDrive 23 Plus, Intelligent Motion Systems, Inc.) placed at the centre of the cage roof. This setup allowed the bird to feed from the spinning feeder, while maintaining its centre of gravity in the same spot, executing a pure yaw turn. A white cardboard square was placed above the hummingbird to offer a contrasting background to the hummingbird body. The hummingbirds were trained to follow the feeder at 30 r.p.m. A stationary hovering flight was recorded using a high speed camera (Fastec Imaging, Troubleshooter $)$ with spatial resolution of $(640 \times 480)$ and a temporal resolution of $1000 \mathrm{fps}$. In this study, a set of videos recorded the hummingbird flight from a ventral view perspective (see Figure 1 ). A total of 91 flight cycles were herein considered. For each wing bird, an average of $X$ wingbeats were computed independently. A complete description of the data herein used were already reported by Altshuler et al. who also captured muscle activation patterns with an electromiography device [4]. The electrical muscle activity was not herein considered since it was out of the scope of this paper, devoted to introduce a fully automatic method for analysis of the kinetic patterns.

Additionally, a controlled experiment was carried out by estimating the angular velocity of a rigid plate perpendicularly coupled to a servo-motor and recorded with a high speed $\ddagger$ where $l$ stands for the left and $r$ for the right 
camera, as shown in Figure $X$. The rotating mechanism used an encoder to control the angular velocity with a temporal resolution of 4096 pulses per revolution. This mechanism was captured with a high-speed camera, $102 \mathrm{fps}$, and a spatial resolution of $640 \times 480$.

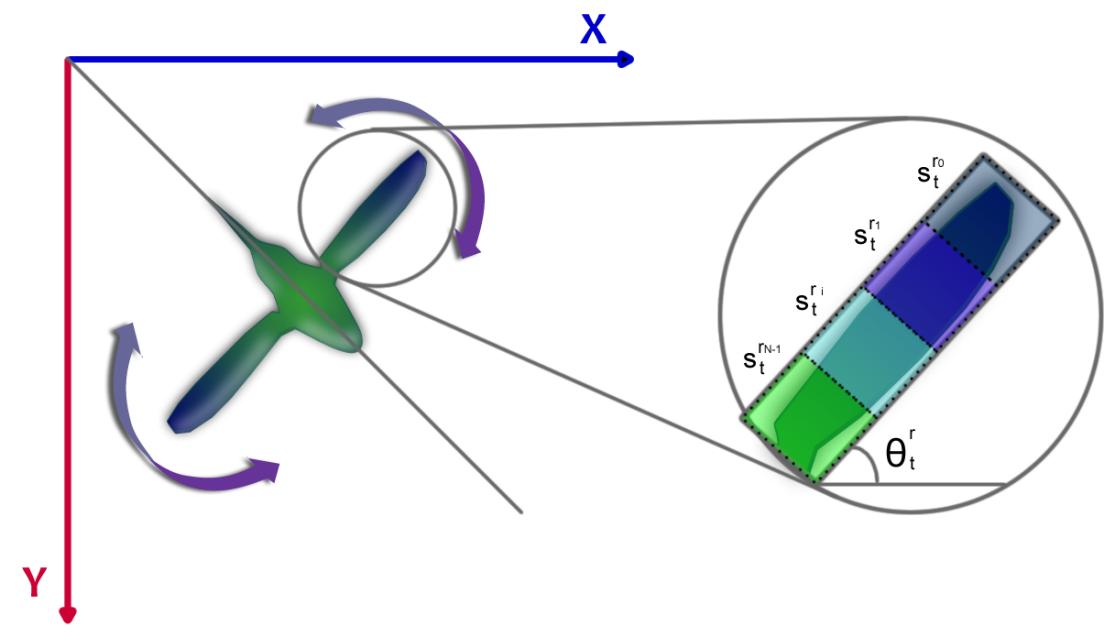

(a)

(b)

Figure 1. In (a) is shown the ventral configuration perspective used to record the hummingbird flight. In (b) is illustrated the computation of the $N$ wing segments using as reference a rotated bounding box

\subsection{Statistical Analyses:}

Statistical Analyses: The downstroke and upstroke were separated and a transition phase was defined as the interval between $40 \%$ and $60 \%$ of the normalized stroke. The t-student approach was used for pair comparisons between different variables, utilizing a mean value for each bird and for each flight phase. A first comparison was carried out between the angular acceleration computed with the proposed approach and manual delinations of the wing. Likewise, the t-student was used for comparison between the right and left wings of the fourt birds.

\section{Results}

The set of motion measures, herein proposed to analyze the hummingbird yaw turn flight, provides a valuable spatio-temporal wing description from local and global perspectives, highlighting the maximal and minimal moments of the angular acceleration and the wing deformation. The introduced analysis framework, allows a direct wing kinematic analysis, promoted by a dense optical flow that facilitates a spatio-temporal characterization of a particular wing region. In other words, this method tracks the whole wing structure and permits continuous local or regional analyses of the wing, much more representative of the wing dynamics than the few classical points of interest marked by an expert. 

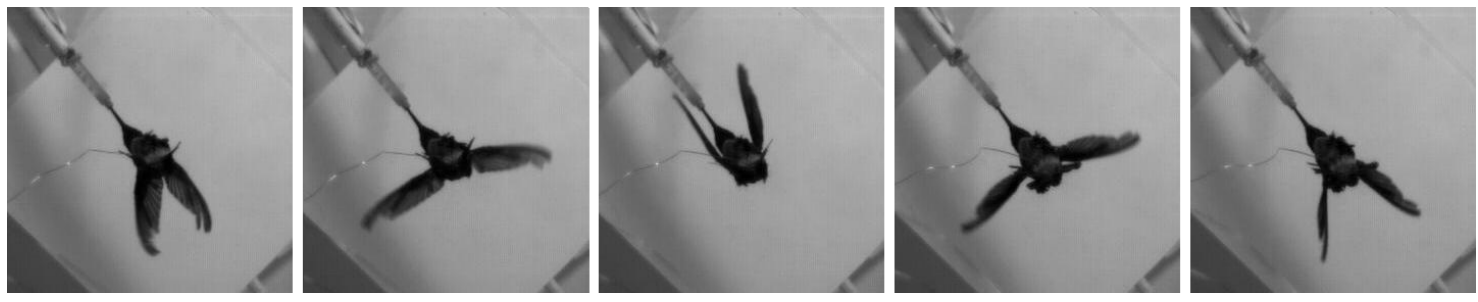

(a) hummingbird flight video sequence
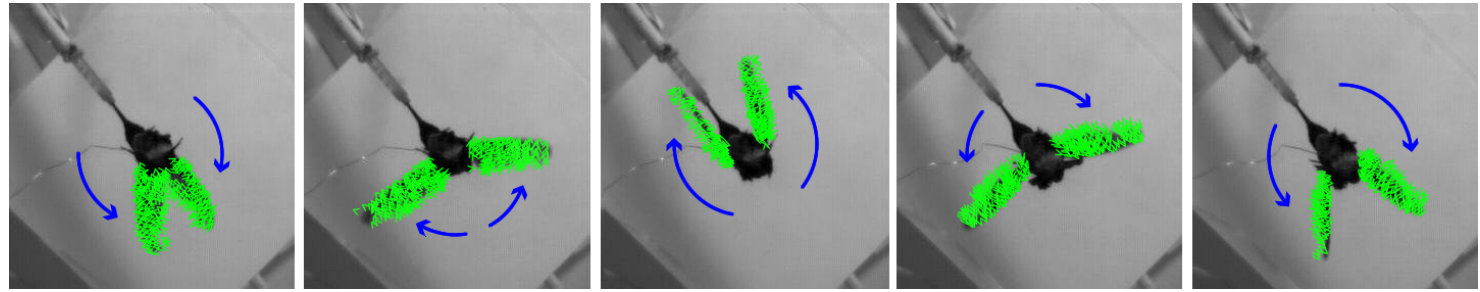

(b) Dense optical flow computation during the hummingbird flight. The optical flow field in each frame it is represented by a set of green arrows.
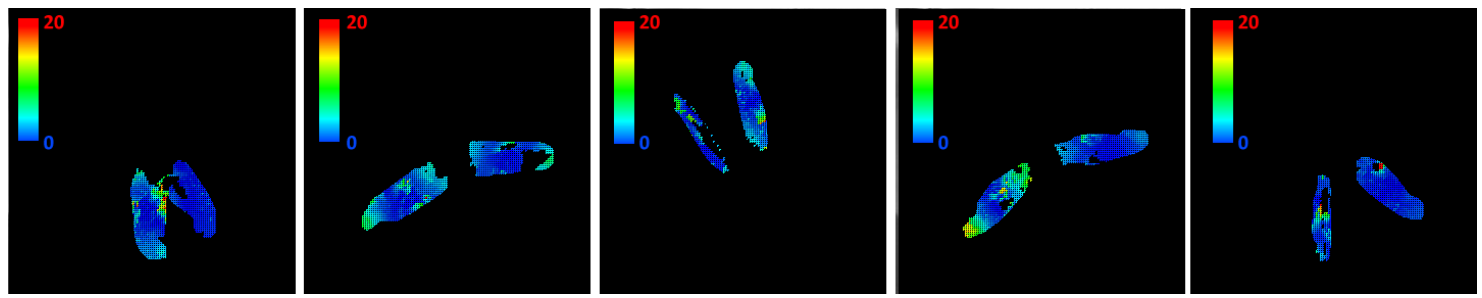

(c) Deformation map obtained from the orientation variance w.r.t to the average orientation in each frame. In this color scale the larger deformation is represented by the red color.

Figure 2. Hummingbird wing motion analysis from a dense optical flow computation.

An accurate estimation of the velocity with an optical flow field would facilitate the extraction of any motion pattern. However, a proper understanding the dynamics along the flight requires a balance between global and local information, a task for which a conventional optical flow is insufficient because it performs pixel matching at a very local level, while the quality of the global information depends on the spatial regularizer. In contrast, the multidimensional dense flow herein implemented, is more appropriate since matching is performed at regional level and no explicit spatial regularization is needed. A typical multidimensional dense flow estimation of the hummingbird flight is shown in Figure 6(b), where the apparent velocity vectors are observed to be mainly within the wings. However these multi scale optical flow advantages, the matching in large scales ( computed with large $\sigma)$ may present some spatial inconsistencies regarding the location of the resultant vectors, for instance the vectors surrounding the wings, yet these vectors do represent actual dynamic patterns. For the experiments presented hereafter, the dense optical flow was parameterized as follows: 7 scales with a $\sigma_{0}=0.3$ and $\sigma_{n+1}=2 \sigma_{n}$, while up to the second order derivatives were used, resulting in a descriptor vector of dimension 42 .

For the controlled experiment, the rotating plate mechanism was triggered for different angular accelerations, ranging from 0.1 to $1 \frac{\mathrm{rad}}{\text { frame }^{2}}$, according to the encoder sensor. Once 
the mechanism started from a pre-defined value, the camera recorded a sequence and a dense optical flow was computed. Figure 3 shows the dense optical flow calculated from a typical sequence of the rigid plate. The dense optical flow without any spatial restriction tracked the plate, clustering the regions with motions. Under a RMSE metric, the proposed approach achieved an averaged accuracy of 87.2 w.r.t to the mechanism sensor.

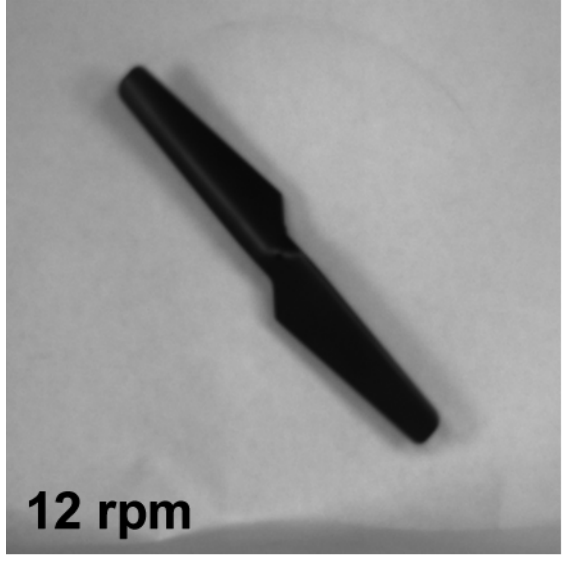

(a)

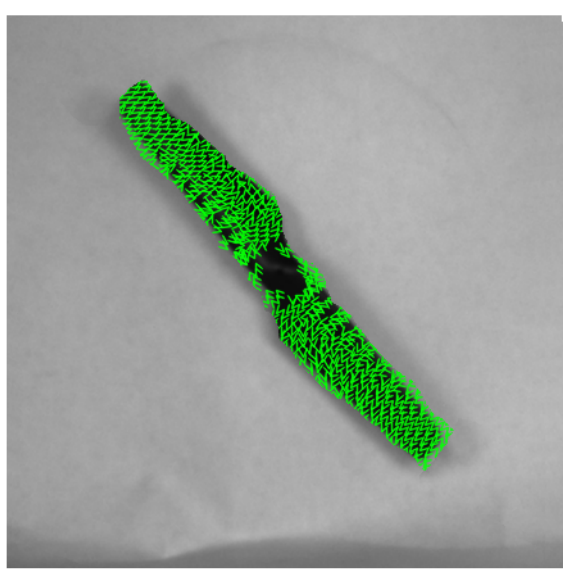

(b)

Figure 3. In (a) the artificial rotating plate mechanism is shown using the proposed approach to test. In (b) a typical dense optical flow computation from the artificial mechanism is illustrated, notice the green arrows represent the speed vectors.

Figure $2 c$ shows the temporal deformation map $Q_{t}(\mathbf{x})$ as a function of time and space $(\mathrm{x}, t)$ obtained during a stroke cycle. The color scale represents the magnitude of the local deformation along the wing. Such deformation maps, estimated from the velocity fields, are totally coherent with the classical bio-mechanical descriptions of the musculoskeletal hummingbird structure: during the first stroke phase (corresponding to the [0 - 20\%] of the temporal serie) [3], [4]. This action produces an initial wing deformation, basically controlled by the proximal wing, as illustrated in the first panel of the deformation map(Figure 2(c)). During the intermediate stroke phase, the wing deformation is propagated to the distal segments, devoid of any skeletal structure, and whose maximal deformation occurs at about $60 \%$ of the stroke, as observed in the mid panel of the Figure 2(c). The final cycle is completed by backwardly extending the wings, as illustrated in the last panel of figure 2(c).

The estimated global angular acceleration $\Omega_{t}$ correlates with the wing deformation and was calculated as the derivative of the averaged orientation of the apparent velocity flow for each wing. Every flight stroke, computed from each video, was normalized to be between $0 \%$ and $100 \%$ and then manually selected and phase-aligned by an expert. The pattern of a cycle was estimated by determining the correlation in the experimental group: for the whole set of 91 recorded sequences and for each instant $t$, a statistical boxplot was computed, within which the red line represents the median of the angular acceleration, the blue lower and upper boxes stand for the spread of data (interquartile range), the maximum and minimum values are the vertical dotted lines and the red asterisks stand for some outliers of the remaining data, as illustrated in Figure 3(a) and 3(b) for the two wings respectively. Of course, the sources 
of error are variable and several measurements were performed to highlight the origin of such variation, namely how much is due to variations among flights, between right and left wings and how much is caused by the new optical flow. The latter question was addressed by comparing the angular acceleration§ obtained either from the optical flow or from a set of manual delineations of the wing. A t-student test showed no significant differences $(p<0.05)$ when comparing the manual and automatic estimations for the four test subjects, 91 flights and the two wings.

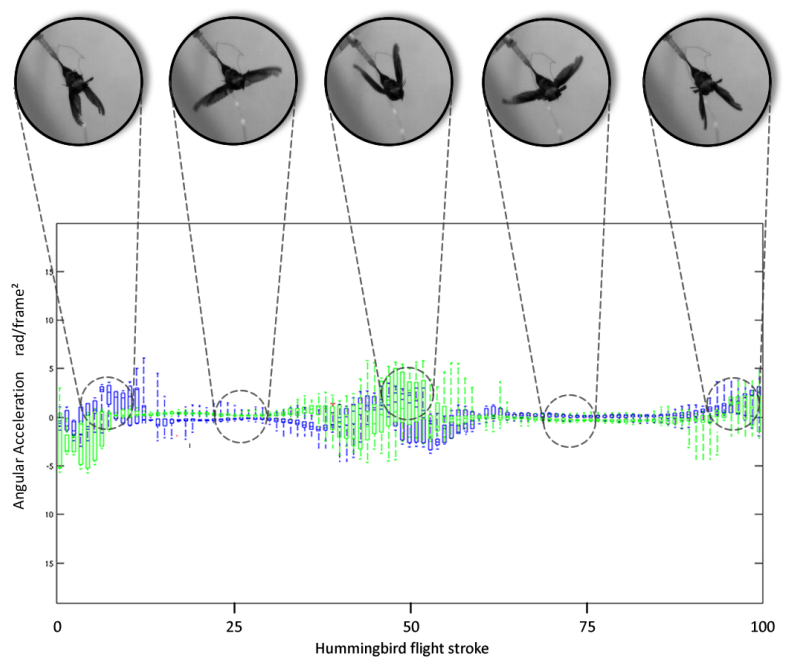

(a) Angular acceleration for the right wing

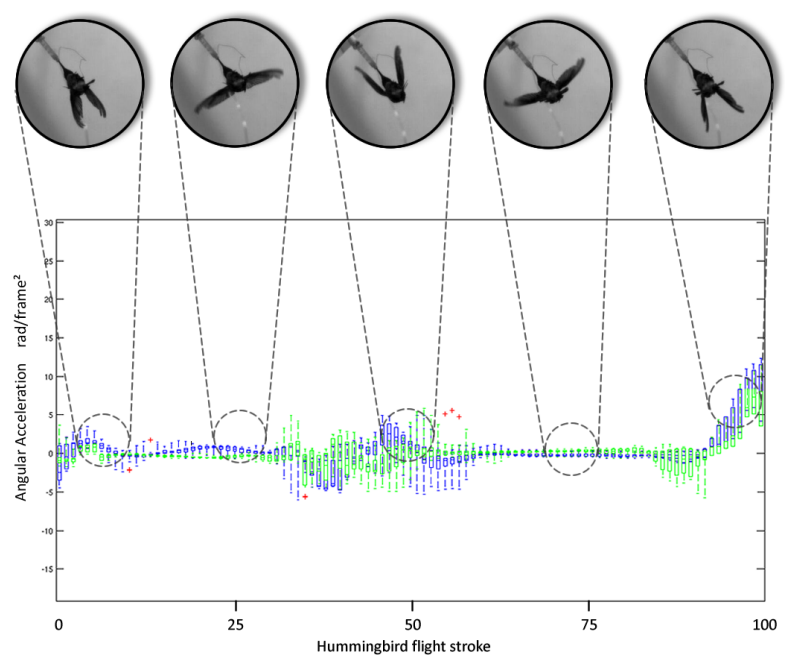

(b) Angular acceleration for the left wing.

Figure 4. The statistical box plot sequences represent the estimated angular acceleration for both wings using the proposed approach within which the red line represents the median of the angular acceleration, the blue lower and upper boxes stand for the spread of data (interquartile range) and the maximum and minimum values are the vertical dotted lines. The green box plot sequence corresponds to the manual estimations performed by an expert.

Regarding the other part of the variability, the temporal normalized stroke follows a very $\S$ the angular acceleration is computed by deriving twice the trajectoty of the Center of Mass during the stroke. 
similar pattern for each of the recorded wings, with minimal variance in the passive phases and larger differences for the active parts of the cycle. On the one hand, the mid part of the temporal normalized stroke shows a larger variance, the transition between the upstroke and downstroke phases. The observed acceleration peak, between the $40 \%$ and $60 \%$ percent of the normalized stroke, represents two complementary actions: an initial relaxation, when the wings move forwardly and join, followed by the muscle contraction required to separate them. During the transition between these two actions, the mean and standard deviation of the angular acceleration were computed for the right, $0.058 \pm 0.469 \frac{\mathrm{rad}}{\mathrm{frame}^{2}}$, and left, $-0.79 \pm 0.526 \frac{\mathrm{rad}}{\text { frame }^{2}}$, wings. A conventional t- student test showed no significant differences for $\alpha=0.05$. However when this analysis was performed individually, that is to say left and right wings of the same bird, significant differences appear (t-student test, $p<0.05$ ) as illustrated in table 1.

On the other hand, the upstroke and downstroke phases correspond to the [10\% - 40\%] and [60\%-90\%] of the temporal normalized stroke, respectively. In such intervals, the flight is basically passive and smooth because motion is mainly driven by the inertia. For the upstroke phase, the mean and standard deviations for the right and left wings were $-0.03 \pm 0.291 \frac{\mathrm{rad}}{\mathrm{frame}^{2}}$ and $0.143 \pm 0.247 \frac{\mathrm{rad}}{\mathrm{frame}^{2}}$ respectively, while the mean and standard deviations were for the right, $-0.025 \pm 0.249 \frac{\mathrm{rad}}{\text { frame }^{2}}$, and left, $-0.015 \pm 0.298 \frac{\mathrm{rad}}{\text { frame }^{2}}$, wings during the the downstroke, differences that were found to be not significant ( $t$-student test and $p<0.05$ ).

Table 1 shows the mean and standard deviation of the estimated angular acceleration, for each bird, stroke phase and specific wing. As expected, during the upstroke and downstroke, the angular acceleration and its standard deviation are relatively small for the left and right wings of the set of experimental birds. In contrast, during the transition phase the averaged angular acceleration and the standard deviation increase. Interestingly, significant differences were found in the transition phase between the right and left wing for the first and fourth bird.

Yet manual annotations are inevitably influenced by the inter subject variability and therefore they are prone to errors, such estimations were herein used as a reference frame of the flight patterns. A comparison of the computed kinematic paths was carried out w.r.t estimated motion trajectories from the expert segmentations. For doing so, each wing was delineated for the complete stroke sequence of every available flight. Then, the global angular acceleration was computed as the second derivative of the Center-of-Mass trajectory of every wing. The resultant mean and standard deviation of the angular acceleration obtained from manual delineations is plotted in Figure 3 (green line), and a close match with the automatic estimations is observed in this graph, a statement supported by a correlation factor of $0.84 \pm 0.12$.

A local angular acceleration profile was computed by dividing the wing into segments, the angular acceleration being regionally estimated for each segment during the flight stroke cycle. Figure 3 shows a typical map acceleration profile, during a cycle and using eight wing segments. Each computed map reveals further details about how the angular acceleration are distributed along the wing, from the proximal to distal regions along the stroke flight. As 


\begin{tabular}{c|cccccc}
\hline & \multicolumn{2}{|c}{ upstroke } & \multicolumn{2}{c}{ downstroke } & \multicolumn{2}{c}{ transition } \\
& Right & Left & Right & Left & Right & Left \\
\hline B.1 & $0.2 \pm 0.75$ & $0.2 \pm 0.2$ & $0.1 \pm 0.11$ & $-0.2 \pm 0.08$ & $-0.4 \pm 0.36^{*}$ & $-0.1 \pm 0.46^{*}$ \\
& & & & & & \\
B.2 & $0.3 \pm 0.06$ & $-0.3 \pm 0.03$ & $-0.1 \pm 0.46$ & $0.1 \pm 0.68$ & $0.3 \pm 0.62$ & $0.1 \pm 0.76$ \\
& & & & & & \\
B.3 & $0.1 \pm 0.28$ & $-0.1 \pm 0.38$ & $0.1 \pm 0.38$ & $-0.1 \pm 0.36$ & $-0.1 \pm 0.44$ & $0.2 \pm 0.37$ \\
B.4 & $-0.7 \pm 0.06$ & $0.8 \pm 0.36$ & $-0.1 \pm 0.03$ & $0.1 \pm 0.05$ & $0.3 \pm 0.46^{*}$ & $-3.3 \pm 0.51^{*}$ \\
\hline
\end{tabular}

Table 1. The mean and standard deviation of the angular acceleration are presented for the right and left wing of each of the four birds. Such analysis is independently performed for each of the different stroke phases. During the upstroke and downstroke phases, the angular acceleration showed not significant differences among the different bird strokes $(t$-student test and $p<0.05$ ) since the the flight is basically passive. In contrast, during the transition phase the averaged angular acceleration and standard deviation increased since the motion is highly dependent of the bird. The significant differences found in this phase (t-student) test, $p<0.05$ are marked with the asterisk.

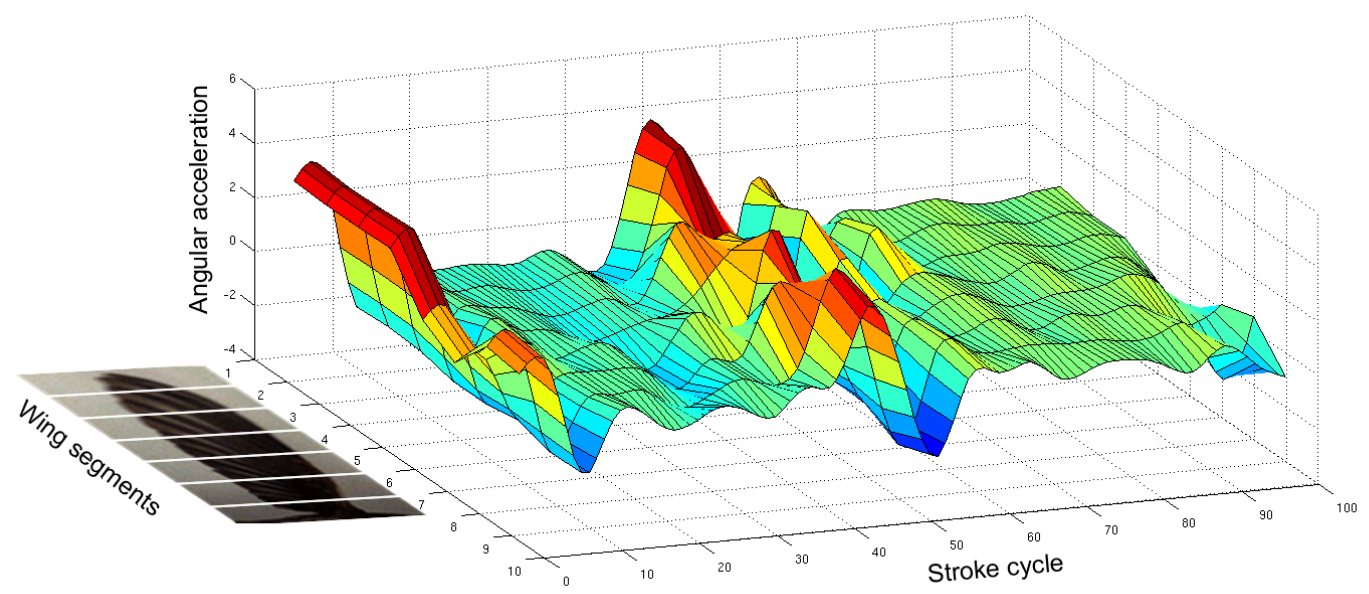

Figure 5. Local angular acceleration profile by the subdivision of the wing in eight segments.

expected, in the first phase of the stroke cycle, the acceleration profile is very similar for the proximal and distal parts. At about a $30 \%$ of the cycle, this pattern changes and the distal part is fully responsible for the observed motion changes. The proximal wing simply leverages the distal motion whose amplitude varies in several mini-cycles to a larger extension at the end of the cycle. From such acceleration map, it was also possible to observe the non linear distribution of the angular acceleration in both axes: wing position and temporal cycle.

Finally, a global wing deformation, during a stroke cycle, was estimated from the whole experimental group $W_{t}^{l}$ (resp. $W_{t}^{r}$ ), and shown in Figure 5. In general, every series of the wing deformation follows a very similar pattern, with a variance whose amplitude is smaller than a $10 \%$ of the spanned wing amplitude. As for other deformation estimations, a statistic boxplot was computed at each time of the cycle, using every recorded video, and again the red 
line represents the sample median while the spread of data is represented by the blue lower and upper quartiles. The larger stroke deformations correlate with the maximum wing effort moments, when motion abruptly rises and there exists a change of direction.

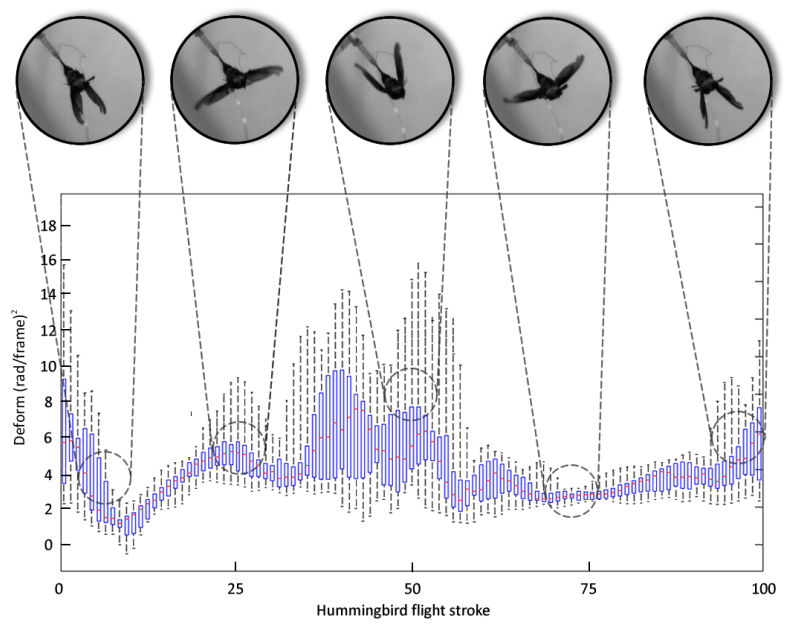

(a) Global wing deformation for the right wing

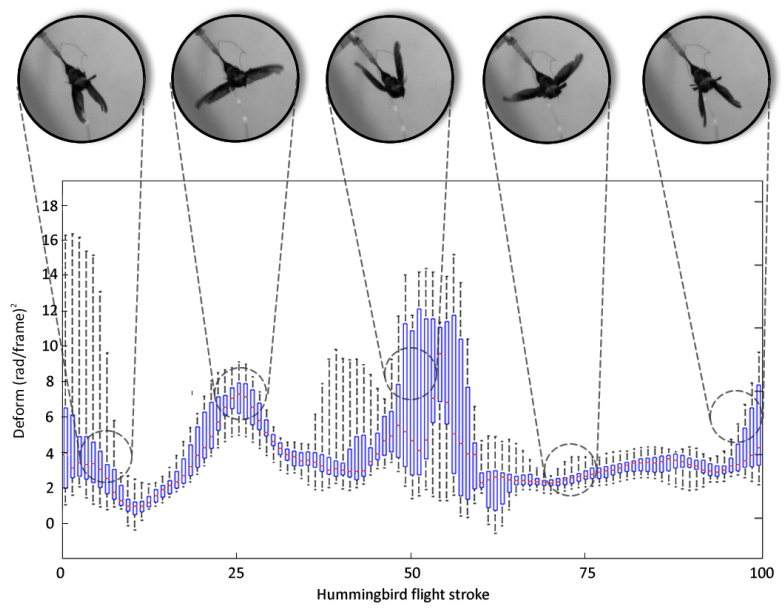

(b) Global wing deformation for the left wing.

Figure 6. The statistical box plot sequences represent the estimated gobal wing deformationn for both wings using the proposed approach

An independent analysis for the three flight stroke phases was also performed for the global deformation patterns. As expected, during the downstroke and upstroke phases, when there exists a relatively relaxed state and motion is almost constant, the wings act like rigid structures with small deformations, principally at the wing borders. The pattern is systematically reproduced by the different wings, with a very small standar deviation (around $\left.1.96\left(\frac{\mathrm{rad}}{\text { frame }}\right)^{2}\right)$. On the other hand, during the transition stroke phase, the deformation increases, as well as the variance. During this phase, the deformation is large because of the rapid wing adaptation to the new change of acceleration direction.

This dense optical flow software was developed in house by the authors and was implemented by authors in $c++$. This particular dense optical flow has been already tested in 
other motion applications [25], [36]

\section{Discussion}

This work introduced a novel approach to perform a complementary kinematic analysis of the hummingbird wing motion, using a multidimensional dense optical flow and without any prior information or any invasive sensor that could alter the natural flight motion gesture. This markerless strategy opens up the possibility of capturing additional spatiotemporal relationships since this is a much more complete representation of the wing dynamic than the classical points of interest that are marked by an expert along a recorded video sequence. While optical flow approaches such as Lukas-Kanade are relatively robustness to noise, the resulting flow basically tracks certain salient features, in particular the edges [39], [40]. Alternatively, global approaches, like Horn and Schunk, are limited to smooth vector fields that capture slow changes but are also more sensible to noise because the flow representation is obtained at the pixel level. A hummingbird flight analysis from this type of dense flows may lose relevant flight features, namely, during the transition between the upstroke and downstroke phases. In contrast, the optical flow herein applied extracts relevant information rather than the apparent location of individual pixels, and therefore ends up by being a suitable and robust tool to determine the fundamental motion wing patterns. textcolorMyColorThe herein proposed approach is able to compute the angular velocity and to estimate the wing deformation during the hummingbird yaw turning using a simple dense velocity field computed from video sequences. Since this particular dense optical flow is basically a computation of the spatial changes at multiple scales by using the different derivatives, this approach results to be very robust to the noise. In addition, the temporal correlation is used to remove different types of illumination noise or artifacts produced by the capturing procedure. The main limitation, in contrast, comes from the number of scales used in terms of computational time. The whole picture is reconstructed by means of a set of kinematic measurements computed from the velocity optical flow field, which was in due turn used to spatially segment and cluster the different wing flow patterns. Angular acceleration and temporal wing deformation measures were herein estimated from local and global perspectives over a set of 91 videos. Quantitative metrics and results showing a low variance of the computed kinematic patterns among the evaluated sample as showed in the variance analysis evaluation.

Flight analyses by means of physical approximations are often based on the study of some temporal sparse biomarkers, and therefore only provide temporal information of very simplified structures, or become statistically insignificant. These approximations in consequence result insufficient or unsuitable towards understanding complex maneuvers, especially when such motions are directly related with temporal non-linear structural deformation. Some computational models partially overcome such simplicity, allow for simulation of several flight patterns by subdividing the wing into simpler parts which are steered by independently parameterized physical models [20]. However, such simulations are usually unrealistic and far from being interpretable, they would hardly discover complicated 
relationships among forces, interactions, neuromotor commands and joint movements that might produce complex motions such as the hang up flight. In contrast, the proposed approach is able to describe convolved kinematic patterns that are spatially spread out along the wing, using exclusively the video information, without prior hypotheses. In this sense, the presented strategy not only can find out reliable motion patterns, but it also may reveal local deformation patterns of the wing .

Rotational patterns are crucial in flight analysis since they allow to study the source of circulation air which is straightforwardly related to the upward and downward forces during the stroke cycle. Furthermore, the angular acceleration, the angle of attack and the span ratio are responsible for controlling the upstroke circulation, the wing orientation and the maintenance of the forward flight [28], [29]. The Global angular acceleration herein estimated presents large gradients during the upstroke and downstroke transitions, a result of the upward and downward wing forces which are responsible for the circulation changes. From a local angular acceleration standpoint, the proposed approach also allows to estimate a regional angular acceleration profile along the wing, thereby discovering not only the phases where the sources of circulation change but also determining which particular wing regions are more involved in the generation of these forces. As illustrated in Figure ??, the main angular acceleration modes are initially concentrated in the proximal wing regions because the shoulder and pectoral muscles drive the forward wing motion, a pattern that is displaced to the distal wing regions when the passive accumulated energy rules the downstroke phase. Although the proposed method is able to capture local motion patterns from the dense optical flow, the spatial accuracy is restricted by the number of scales and the standard deviation used to convolve each frame. For instance, when the scales are obtained by convolving Gaussians with large sigmas, results would be more robust to noise from a motion but less accurate spatially. A proper balance between the noise control and the spatial accuracy is part of the method tuning as to obtain coherent results.

On the other hand, the stunning deformations of the wing are responsible for much of the salient abilities of hummingbirds such as their stability and relative conservation of the orientation, even if they are forced to change constantly their acceleration patterns [42], [41]. In addition, localized wing deformations may produce relative point circulation vortices that induce a non linear pattern along the wing structure and therefore completely alter the hummingbird flight smoothness [33]. In the proposed strategy, deformation wing patterns were locally estimated along the wing as the relationship between each local orientation w.r.t an average orientation, resulting in a wing map of deformation for every stroke. Main wing deformations are commonly localized along the proximal and distal segments while the largest deformation is observed when the wing acceleration is maximum, i.e., during the downstroke and upstroke transitions (see Figure 5).

Recent methodological advances have improved our comprehension of the most complex hummingbird maneuvers and their intrinsic mechanisms, for instance, aerodynamic studies using PIV have shown an asymmetric distribution of the lift forces during the stroke, probably caused by unknown micro-mechanisms like the stall and leading-edge vortices [15]. New analysis strategies are then required to approach such local and non linear 
phenomena whose role is yet an open problem. The proposed approach herein introduced is a global-to-local analysis tool that complements the existent methods, with the benefit of methodological simplicity. Likewise, the two variables herein estimated, angular acceleration and wing deformation, were locally and globally computed, opening thereby the possibility of conducting more subtle studies that discover the wing regions that contribute to every step of the hummingbird yaw turn.

The kinematic patterns herein reported were captured via a single camera from only a ventral perspective, however this analysis can be directly extended to analyze the complementary dynamic patterns in the other planes. Likewise, the proposed approach can easily adapted to a $3 \mathrm{D}$ reconstruction by using multiple calibrated cameras. The proposed approach may also able to locally compute force and torque patterns at the different wing segments by directly relating the angular acceleration with the segmented anatomical structured. Finally, this method can be used to automatically segment the hummingbird flight strokes in sequences of video, highly reducing inter and intra expert variability. In addition, the proposed approach can be potentially applied even when the animal is moving along the scene, since once the dense optical flow tracks the main regions in motion, this bounded region may be followed and characterized.

\section{Conclusions}

This work has presented a novel approach to quantify the yaw turn hummingbird motion, using a dense optical flow computed from video sequences. A main contribution is the markerless flight analysis framework that estimates two relevant kinematic hummingbird variables i.e., the angular acceleration and wing deformation. The proposed method opens up an actual possibility of understanding more complex yaw turn phenomena. Future works include the study and development of a robust method that temporally relates the kinematic dense flow description with force measurements generated by flapping, under actual anatomical and mass parameters.

\section{Acknowledgements}

The data used in the present investigation was kindly provided by the department of Zoology of the University of British Columbia. We wish to thank Dr Douglas Altshuler, Elsa Quicazan, and Dimitri Skandalis for their discussions that highly enriched the present work.

\section{References}

[1] E. de Margerie, J. B. Mouret, S. Doncieux, and J.A. Meyer. Artificial evolution of the morphology and kinematics in a flapping-wing mini uav Bioinspir. Biomim. (2) 65-82 (2007)

[2] D. Altshuler and R. Dudley. The ecological and evolutionary interface of hummingbird flight physiology J. Exp. Biol. (205) 2325-2336 (2002) 
[3] Douglas L Altshuler and Kenneth C Welch and Brian H Cho and Danny B Welch and Amy F Lin and William B Dickson and Michael H Dickinson Neuromuscular control of wingbeat kinematics in Anna's hummingbirds (Calypte anna). The Journal of Experimental Biology. (215) 4070-4084 (2010)

[4] Douglas L. Altshuler, Elsa M. Quicazán-Rubio, Paolo S. Segre, and Kevin M. Middleton Wingbeat kinematics and motor control of yaw turns in Anna's hummingbirds (Calypte anna) The Journal of Experimental Biology. (213) 2507-2514 (2012)

[5] Hedrick, T. L. and Biewener, A. A. Low speed maneuvering flight of the rose-breasted cockatoo (eolophus roseicapillus) i. kinematic and neuromuscular control of turning. The Journal of Experimental Biology. (210) 1897-1911 (2007)

[6] Hedrick, T. L., Usherwood, J. R. and Biewener, A. A. Low speed maneuvering flight of the rose-breasted cockatoo (eolophus roseicapillus). ii. inertial and aerodynamic reorientation. The Journal of Experimental Biology. (210) 1912-1924 (2007)

[7] Warrick, D. R., Dial, K. P. and Biewener, A. A. Asymmetrical force production in the maneuvering flight of pigeons. The Auk. 115(4) 916-928 (1998)

[8] Warrick, D. R., Bundle, M.W., and Dial K. P. Bird maneuvering flight: blurred bodies, clear heads. Integrative and Comparative Biology. 42 141-148 (2002)

[9] Warrick, D.R. and M. Dickinson The aerodynamics of hummingbird flight. American Institute of Aeronautics and Astronautics (2007)

[10] Fry R., S. N. and M. H. Dickinson. The aerodynamics of free-flight maneuvers in drosophila. Science, 300(5618), 495-498 (2003)

[11] Dawson, J. W. Dawson-Scully and R. Robertson. Forewing asymmetries during auditory avoidance in flying locusts The Journal of Experimental Biology. 200(17) 2323-2335 (1997)

[12] , Ortega-Jimenez VM, Dudley R. Source Aerial shaking performance of wet Annaś hummingbirds. Journal of The Royal Society Interface. 9(70) 1093-1099 (2012)

[13] Simon M. Walker and Adrian L. R. Thomas, and Graham K. Taylor Deformable wing kinematics in freeflying hoverflies Journal of The Royal Society Interface. 7(42) 131-142 (2010)

[14] Straw, Andrew D. and Branson, Kristin and Neumann, Titus R. and Dickinson, Michael H. Multi-camera Realtime 3D Tracking of Multiple Flying Animals Journal of The Royal Society Interface. 8(56) 395-409 (2011)

[15] Warrick, D. R., Tobalske, B. W., and Powers, D. R. Aerodynamics of the hovering hummingbird Nature. 435(7045) 1094-1097 (2005)

[16] Bomphrey, Richard J. Advances in Animal Flight Aerodynamics Through Flow Measurement Evolutionary Biology. 39(1) 1-11. (2011)

[17] Adrian, Ronald J. Particle-Imaging Techniques for Experimental Fluid Mechanics Annual review of fluid mechanics, 23(1), 261-304 (1991)

[18] Shyy W. and H. Aono and S.K. Chimakurthi and P. Trizila and C.-K. Kang and C.E.S. Cesnik and H. Liu Recent progress in flapping wing aerodynamics and aeroelasticity Progress in Aerospace Sciences, 46(7), 284-327 (2010)

[19] Bos, F. M. and Lentink, D. and Van Oudheusden, B. W. and Bijl, H. Influence of wing kinematics on aerodynamic performance in hovering insect flight Journal of fluid mechanics, 594(1), 341-368 (2008)

[20] Zongxian Liang and Haibo Dong Computational Analysis of Hovering Hummingbird Flight In 48th AIAA aerospace sciences meeting including the new horizons forum and aerospace exposition. 2010-555 (2010)

[21] Wang, Z. J. and Birch, J. M. and Dickinson, M. H. Unsteady forces and flows in low Reynolds number hovering flight: two-dimensional computations vs robotic wing experiments. Journal of Experimental Biology, 207(3), 449-460 (2004)

[22] Altshuler, Douglas L. and Dudley,Robert and Ellington,Charles P. Aerodynamic forces of revolving hummingbird wings and wing models Journal of zoology, 264(4), 327-332. (2004)

[23] Shyy, W. and Lian, Y. and Tang, J. and Liu, H. and Trizila, P. and Stanford, B. and Bernal, L. and Cesnik, C. and Friedmann, P. and Ifju, P. Computational aerodynamics of low Reynolds number plunging, pitching and flexible wings for MAV applications Acta Mechanica Sinica, 24(4), 351-373.(2008)

[24] Wu, P. and Stanford, B.K. and Sallstrom, E. and Ukeiley, L. and Ifju, P.G. Computational aerodynamics 
of low Reynolds number plunging, pitching and flexible wings for MAV applications Bioinspiration and Biomimetics. Acta Mechanica Sinica, 24(4), 351-373 (2008)

[25] Antoine Manzanera Local Jet Feature Space Framework for Image Processing and Representation In International Conference on Signal Image Technology and Internet Based Systems (SITIS'11).261-268 (2011)

[26] Bentley, Jon Louis Multidimensional binary search trees used for associative searching Commun. ACM, 18(9), 509-517 (1975)

[27] L. Florack, B. Ter Haar Romeny, M. Viergever, and J. Koenderink The Gaussian scale-space paradigm and the multiscale local jet International Journal of Computer Vision. 18(1) 61-75. (1996)

[28] Dickinson, Michael H. and Lehmann, Fritz-Olaf and Sane, Sanjay P. Wing Rotation and the Aerodynamic Basis of Insect Flight Science. 284(5422), 1954-1960 (1999)

[29] Tobalske, Bret W. Biomechanics of bird flight. Journal of Experimental Biology. 210(18), 3135-3146. (2007)

[30] A. Verri and F. Girosi and V. Torre Mathematical properties of the two-dimensional motion field: from singular points to motion parameters J. Opt. Soc. Am. A. 6(5), 698-712 (1989)

[31] Higgins, Longuet H. C. and Prazdny, K. The Interpretation of a Moving Retinal Image Proceedings of the Royal Society of London. Series B, Biological Sciences. 208(1173), 385-397. (1980)

[32] Altshuler, Douglas L., and Robert Dudley. The ecological and evolutionary interface of hummingbird flight physiology. Journal of Experimental Biology. 205(16), 2325-2336. (2002)

[33] Hedrick, T. L., Tobalske, B. W. and Biewener, A. A. Estimates of circulation and gait change based on a three-dimensional kinematic analysis of flight in cockatiels (Nymphicus hollandicus) and ringed turtledoves (Streptopelia risoria). J. Exp. Biol. 205, 1389-1409 (2002)

[34] Ristroph, L., Berman, G. J., Bergou, A. J., Wang, Z. J. and Cohen, I. Automated hull reconstruction motion tracking (HRMT) applied to sideways maneuvers of free-flying insects. J. Exp. Biol. 212, 13241335.(2009).

[35] Steven N. Fry, Rosalyn Sayaman, Michael H. Dickinson The aerodynamics of free-flight maneuvers in Drosophila. Science 300, 495-498.

[36] Fabio Martínez; Antoine Manzanera; Eduardo Romero Representing activities with layers of velocity statistics for multiple human action recognition in surveillance applications Proc. SPIE. 9026, (2014).

[37] Berthold K. P. Horn and Brian G. Schunck. Determining optical flow. Artificial Intelligence. 17:185-203, 1981

[38] Aubert, G., Deriche, R., and Kornprobst, P. Computing optical flow via variational techniques. SIAM Journal on Applied Mathematics, 60(1), 156-182. 1999

[39] Lucas B. and Kanade T. An iterative image registration technique with an application to stereo vision. Proceedings of Imaging Understanding Workshop, 121-130. 1981

[40] Bouguet, J. Y. Pyramidal implementation of the affine lucas kanade feature tracker description of the algorithm Intel Corporation, 5. 2001

[41] Lehmann, F. O., Gorb, S., Nasir, N., and Schutzner, P. Elastic deformation and energy loss of flapping fly wings. The Journal of experimental biology. 214(17), 2949-2961. 2011

[42] Ennos, A. R. The kinematics and aerodynamics of the free flight of some Diptera. The Journal of experimental biology. 142, 49-85. 1989

[43] Keennon M, Klingebiel K, Won $\mathrm{H}$ and Andriukov A. Development of the nano hummingbird: a tailless flapping wing micro air vehicle 50th AIAA Aerospace Sciences Meeting. 0588, 1-24. 2012

[44] Sapir, Nir, and Robert Dudley Backward flight in hummingbirds employs unique kinematic adjustments and entails low metabolic cost The Journal of experimental biology. 215, 3603-3611. 2012

[45] Hedrick, T. L., Cheng, B., and Deng, X Wingbeat time and the scaling of passive rotational damping in flapping flight Science. 324(5924), 252-255. 2009

[46] Walker, J. A. Estimating velocities and accelerations of animal locomotion: a simulation experiment comparing numerical differentiation algorithms. The Journal of Experimental Biology, 201(7), 981-995.

[47] Bansmer, S., Radespiel, R., Unger, R., Haupt, M., and Horst, P Experimental and numerical fluid-structure analysis of rigid and flexible flapping airfoils AIAA journal, 48(9), 1959-1974. 2010. 